\title{
Length-weight relationships of eighteen species of freshwater fishes from Panchet Reservoir in Ganges basin, Jharkhand, India
}

\author{
K. M. SANDHYA ${ }^{1,3}$, GUNJAN KARNATAK ${ }^{1}$, LIANTHUAMLUAIA ${ }^{1}$, UTTAM KUMAR \\ SARKAR $^{1}$, SUMAN KUMARI ${ }^{1}$, P. MISHAL ${ }^{1}$, VIKASH KUMAR ${ }^{1}$, DEBABRATA PANDA ${ }^{2}$, \\ YOUSUF ALI ${ }^{1}$ AND BABLU NASKAR ${ }^{1}$ \\ ${ }^{1} I C A R$ - Central Inland Fisheries Research Institute, Barrackpore - 700 120, West Bengal, India \\ ${ }^{2} I C A R$-Central Institute of Freshwater Aquaculture, Bhubaneswar - 751 002, Odisha, India \\ ${ }^{3}$ ICAR-Central Institute of Fisheries Technology, Willingdon Island, Kochi - 682 029, India \\ e-mail: sandhyafrm@gmail.com
}

\begin{abstract}
The present study describes the length-weight relationships (LWRs) of 18 fish species from a large tropical reservoir, Panchet, in the Damodar River basin, one of the main tributary of the largest river Ganga in India. A total of 2419 individuals represented by 18 species belonging to 9 families were sampled between November 2014 and June 2016. The $b$ values ranged from 2.469 for Trichogaster chuna to 3.428 for Ailia coila. All the regressions were highly significant ( $<<0.001$ ). The results revealed positive allometric growth for seven species $(b>3, p<0.05)$, negative allometric growth for seven species $(b<3, p<0.05)$ and isometric growth for four species $(b=3, p>0.05)$. This study represents the first reference on the lengthweight relationship of Trichogaster chuna from a reservoir ecosystem. This is the first report on LWRs of five fish species viz., Puntius terio, Pethia conchonius, Sperata seenghala, Ailia coila and Trichogaster chuna from an Indian reservoir. This study provides basic data for future stock assessment studies and management programmes from Panchet Reservoir as well as for complementing the comparisons of LWRs from other ecosystems.
\end{abstract}

Keywords: Growth, Length-weight relationships, Panchet, Reservoir, Stock assessment

\section{Introduction}

Length-weight relationship (LWRs) has extensively been used in fisheries research as this enables the conversion of weight of fishes when only their size is known; indicate the type of weight gain and helps formulating management programmes for exploitation of commercial species (Le Cren, 1951; Bolger and Connolly, 1989; Pinheiro and Fiscarelli, 2009). Moreover, this tool also facilitates morphometric comparison between species and populations and life history comparisons between regions (Weatherley and Gill, 1987; Petrakis and Stergeou, 1995; King, 1996; Goncalves et al., 1997). The LWR is also used to define a population, where fish length is measured and the predicted average weight is assigned to all fish in a given length group (Oscoz et al., 2005). This is faster and more convenient than weighing fish individually, especially when large numbers of live fishes are sampled. In fishery biology studies, LWRs are required for conversion of growth-in-length equations to growth-in-weight for using in stock assessment models and to estimate stock biomass from limited sample sizes (Basusta et al., 2013). It is frequently used to track seasonal changes in fish growth (Richter et al., 2000).
Most of the studies in Indian reservoirs are mainly restricted to ecological aspects and fisheries management strategies through fingerling stocking (Sarkar et al., 2018; Lianthuamluaia et al., 2019). LWR studies from tropical inland waters are reported mostly from rivers. Such studies are scarce from Indian reservoirs which form an important inland fisheries resource in the country (Hassan et al., 2017; Sarkar and Mishal, 2017). Present study describes the length-weight relationships of 18 fish species from a tropical large reservoir, Panchet in India comprising commercially important species as well as some of the small indigenous fishes. Only a few fish faunal studies have been reported from this reservoir; mostly limited to catch, fish diversity and LWRs of very few species (Sarkar and Banerjee, 2010; Sandhya et al., 2016, 2017, 2019; Suman et al., 2018). Information on the lengthweight relationship of Puntius terio, Pethia conchonius, Sperata seenghala, Ailia coila and Trichogaster chuna from Indian reservoirs is lacking. Hence the results from this study will provide basic data which would be useful for stock assessment and fisheries management of the species studied as well as for comparisons of LWRs from other ecosystems. 


\section{Materials and methods}

Panchet, a large tropical reservoir along the basin of the river Damodar, one of the main tributary of river Ganga, the largest river in India. Data were collected between November 2014 and June 2016 from Panchet

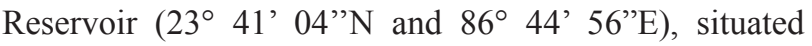
in Dhanbad District in Jharkhand. Fish samples were collected bimonthly using gillnets of mesh size ranging from $25-120 \mathrm{~mm}$ and seine nets of mesh size 10-20 mm from various landing sites including upstream, middle and downstream zones of the reservoir. Fishes were identified to the species level and total length was measured to the nearest $0.1 \mathrm{~cm}$ and weighed individually with an accuracy of $0.01 \mathrm{~g}$. The length-weight relationship was derived using the equation $\mathrm{W}=\mathrm{aL}^{\mathrm{b}}$ where $\mathrm{W}=$ weight of fish in $\mathrm{g}$; $\mathrm{L}=$ length of fish in cm; ' $\mathrm{a}$ ' and ' $\mathrm{b}$ ' are intercept and slope of the regression line respectively (Ricker, 1973). The log-log plots of length and weight of all fishes were made before regression analysis and outliers were removed following the recommendations of Froese (2006). Values of $a$ and $b$ were estimated by regression analysis based on $\log$ values: $\log \mathrm{W}=\log \mathrm{a}+\mathrm{b} \log \mathrm{L}$. Box-Whiskers plots were prepared to describe the distribution of $b$ values. The degree of association between the variables was computed by the determination coefficient, $r^{2}$. The Student's t test was used to test whether the estimated $b$ value was significantly different from the isometric value of 3 at $5 \%$ significance level $(\mathrm{p}<0.05)$ (Sokal and Rohlf, 1987). Comparison between the estimated values of $b$ and respective critical values allowed the determination of statistical significance of $b$ values and their inclusion in the isometric range $(b=3)$ or allometric ranges, i.e., positive allometric $(b>3)$ or negative allometric $(b<3)$.

\section{Results}

The sample size, minimum and maximum lengths and weights, parameters of length-weight relationships, standard error of $b$ values, coefficient of determination $\left(\mathrm{r}^{2}\right)$ and growth type are shown in Table 1. Box-Whiskers plot showing distribution of $b$ values is given in Fig. 1. A total of 2419 individuals represented by 18 species belonging to 9 families were sampled. The most diverse family was Cyprinidae with 6 species. The $b$ values ranged from 2.469 for Trichogaster chuna to 3.428 for Ailia coila. The median value of $b$ was 3.996 and $50 \%$ of $b$ values ranged between 2.832 and 3.130 (Fig. 1). All regressions were highly significant $(\mathrm{p}<0.001)$ with the co-efficient of determination $\left(\mathrm{r}^{2}\right)$ ranging from 0.908 for Parambassis lala to 0.998 for Oreochromis niloticus. The coefficient of determination was greater than 0.95 for 17 species and the remaining one species had $\mathrm{r}^{2}$ of the order of 0.90 . The results revealed positive allometric growth for seven species $(b>3, p<0.05)$, negative allometric growth for

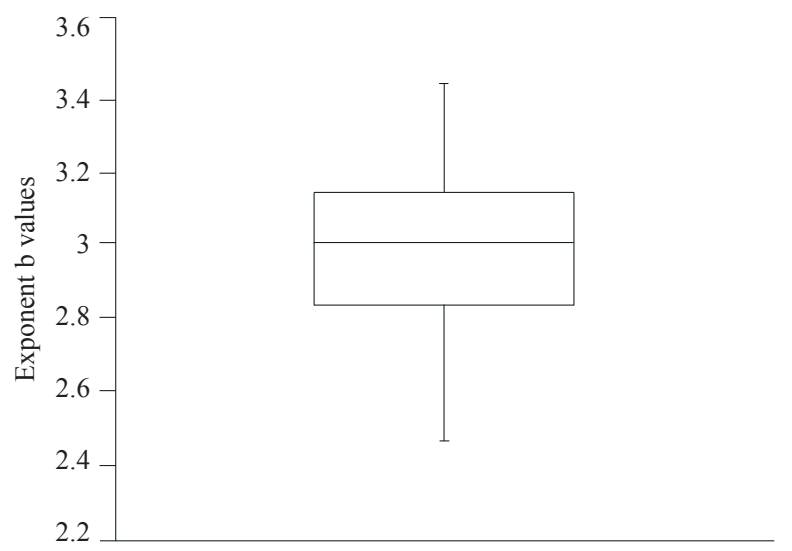

Fig. 1. Box-Whiskers plot of the exponent $b$ values in the length-weight relationships of 18 species from Panchet Reservoir. Central box covers $50 \%$ of data values, the horizontal lines show the median values and vertical lines show the range of values

seven species $(b<3, p<0.05)$ and isometric growth for four species $(b=3, p>0.05)$.

\section{Discussion}

This is the first report on the length-weight relationship of the species Trichogaster chuna from a reservoir ecosystem. The findings from this study also form first report on length-weight relationship parameters for 5 fish species viz., Puntius terio, Pethia conchonius, Sperata seenghala, Ailia coila and Trichogaster chuna from an Indian reservoir as most of the reports are from rivers and wetlands (Table 2). Of these, A. coila is in Near Threatened category (IUCN, 2017) which further highlights the importance of the study with respect to conservation aspects.

The $b$ values in LWRs determine the growth pattern of the fish species. When $b$ is equal to 3 or close to 3 , growth in the fish is said to be isometric i.e., fish maintain their form with increasing length (Froese, 2006). Similarly when $b$ is far less or greater than 3 , growth in the fish is allometric i.e., the fish becomes thinner or fatter with increase in length (Froese, 2006). In our study, all species had $b$ values within the expected range of 2.50-3.50 (Pauly and Gayanilo, 1997; Froese, 2006). All LWRs reported were highly significant with all $r^{2}$ values $>0.900$ and they were compared with the available literatures from various ecosystems. When comparing with studies from reservoir, for species such as Labeo calbasu, Puntius sophore, Pethia phutunio, Chanda nama, Parambassis lala, Parambassis ranga, Notopterus notopterus and Channa punctata, the growth type reported in the present study is similar to the findings from Hirakud Reservoir in Odisha, India (Subodh et al., 2018). Similarly, growth pattern of Oroechromis niloticus 
Table 1. Descriptive statistics and estimated parameters of length-weight relationship of 18 fish species from Panchet Reservoir

\begin{tabular}{|c|c|c|c|c|c|c|c|c|c|c|}
\hline \multirow{2}{*}{ Species } & \multirow[b]{2}{*}{$\mathrm{N}$} & \multicolumn{2}{|c|}{ Length $(\mathrm{cm})$} & \multicolumn{2}{|c|}{ Weight (g) } & \multicolumn{3}{|c|}{ Regression parameters } & \multirow[b]{2}{*}{$\mathrm{r}^{2}$} & \multirow{2}{*}{ Growth type } \\
\hline & & Min & Max & Min & Max & $\mathrm{a}$ & $\mathrm{b}$ & S.E. (b) & & \\
\hline \multicolumn{11}{|l|}{ Cyprinidae } \\
\hline Amblypharyngodon mola & 84 & 2.5 & 9.4 & 0.19 & 8.03 & 0.0120 & 2.950 & 0.048 & 0.983 & I \\
\hline Labeo calbasu & 92 & 4.8 & 48.5 & 2 & 1387.2 & 0.0122 & 3.009 & 0.077 & 0.986 & I \\
\hline Puntius terio & 55 & 2.6 & 5.8 & 0.3 & 3.01 & 0.0115 & 3.147 & 0.054 & 0.974 & $\mathrm{~A}+$ \\
\hline Puntius sophore & 138 & 3.4 & 10.5 & 0.5 & 19.2 & 0.0094 & 3.223 & 0.033 & 0.987 & $\mathrm{~A}+$ \\
\hline Pethia conchonius & 145 & 3.6 & 6.7 & 0.6 & 4.5 & 0.013 & 3.077 & 0.033 & 0.977 & $\mathrm{~A}+$ \\
\hline Pethia phutunio & 147 & 1.8 & 3.6 & 0.12 & 0.64 & 0.0219 & 2.677 & 0.036 & 0.950 & A - \\
\hline \multicolumn{11}{|l|}{ Ambassidae } \\
\hline Chanda nama & 454 & 2.2 & 6.7 & 0.1 & 2.9 & 0.0114 & 2.827 & 0.052 & 0.960 & A - \\
\hline Parambassis lala & 330 & 1.7 & 3.7 & 0.12 & 0.78 & 0.0276 & 2.526 & 0.041 & 0.908 & A - \\
\hline Parambassis ranga & 305 & 1.6 & 8.9 & 0.1 & 10.9 & 0.0179 & 2.946 & 0.017 & 0.990 & A - \\
\hline \multicolumn{11}{|l|}{ Bagridae } \\
\hline Sperata seenghala & 85 & 12.1 & 85.8 & 9.84 & 1250 & 0.0078 & 2.848 & 0.056 & 0.988 & A - \\
\hline Sperata aor & 63 & 8.5 & 65 & 4 & 1231 & 0.0124 & 2.743 & 0.049 & 0.995 & A - \\
\hline \multicolumn{11}{|l|}{ Gobidae } \\
\hline Glossogobius giuris & 158 & 2.4 & 13.8 & 0.16 & 25.14 & 0.0091 & 2.983 & 0.065 & 0.989 & I \\
\hline \multicolumn{11}{|l|}{ Schilbeidae } \\
\hline Ailia coila & 121 & 7.2 & 15.8 & 0.86 & 14 & 0.0012 & 3.428 & 0.039 & 0.968 & $\mathrm{~A}+$ \\
\hline \multicolumn{11}{|l|}{ Cichlidae } \\
\hline Oreochromis niloticus & 57 & 10 & 44 & 17 & 1784 & 0.0146 & 3.130 & 0.039 & 0.998 & $\mathrm{~A}+$ \\
\hline \multicolumn{11}{|l|}{ Notopteridae } \\
\hline Notopterus notopterus & 60 & 4.5 & 31 & 0.79 & 283.3 & 0.0046 & 3.202 & 0.064 & 0.989 & $\mathrm{~A}+$ \\
\hline \multicolumn{11}{|l|}{ Osphronemidae } \\
\hline Trichogaster chuna & 55 & 2.5 & 13.7 & 0.2 & 25.4 & 0.0387 & 2.469 & 0.082 & 0.986 & A - \\
\hline \multicolumn{11}{|l|}{ Siluridae } \\
\hline Ompok bimaculatus & 62 & 11.6 & 31.7 & 9.1 & 170.5 & 0.0038 & 3.131 & 0.042 & 0.982 & $\mathrm{~A}+$ \\
\hline \multicolumn{11}{|l|}{ Channidae } \\
\hline Channa punctata & 68 & 3.6 & 23.1 & 0.5 & 135.9 & 0.0103 & 3.025 & 0.035 & 0.992 & I \\
\hline
\end{tabular}

$\mathrm{N}=$ sample size; $\mathrm{a}$ and $\mathrm{b}=$ parameters of length-weight relationship; S.E. $=$ Standard error; $\mathrm{r}^{2}=$ Co efficient of determination; $\mathrm{I}=$ Isometry; $\mathrm{A}^{+}=$Positive
allometry; $\mathrm{A}^{-}=$Negative allometry

reported from Ero Reservoir, Nigeria is in conformity with the present study (Adebola et al., 2016). In contrary, the species like Amblypharyngodon mola and Glossogobius giuris showed isometric growth in the present study whereas it was reported as negative allometric growth from Hirakud Reservoir. Also in our study, we observed positive allometry for Ompok bimaculatus whereas it was isometric growth from Hirakud Reservoir (Subodh et al., 2018). Some variations could be attributed to the combination of one or more factors such as number of specimens examined, area/seasonal effect, habitat, degree of stomach fullness, gonadal maturity, sex, health and general fish condition, preservation technique and differences in the observed length ranges of the specimens caught, ecological conditions of the habits or the physiology of animals (Le Cren, 1951; Wootton, 1999). Similarly, the parameter a may vary seasonally, daily and/or between different habitats whereas the parameter $b$ which is characteristic of species usually does not vary significantly throughout the year (Bagenel and Tesch, 1978). Since samples have been collected over an extended period of time, these data are not representative of a particular season or time of the year and should be considered as mean annual values for comparisons as suggested by Goncalves et al. (1997).

The present study is the first to report on the length-weight relationship of Trichogaster chuna from a reservoir ecosystem as the previous report is from a wetland ecosystem (Borah et al., 2017). Most of the previous studies on the LWRs of the 18 species studied are from rivers, wetlands and a few from reservoirs. Hence the results in this study represented additional contribution to the available LWR data from other geographical areas and will be useful for the comparisons of LWRs from different ecosystems. To the best of our knowledge, this study presents the preliminary references on the LWR of 18 fish species from Panchet Reservoir in India. Information of individual body length-weight relationships in the 
Table 2. Length-weight relationships of freshwater fish species from previous studies

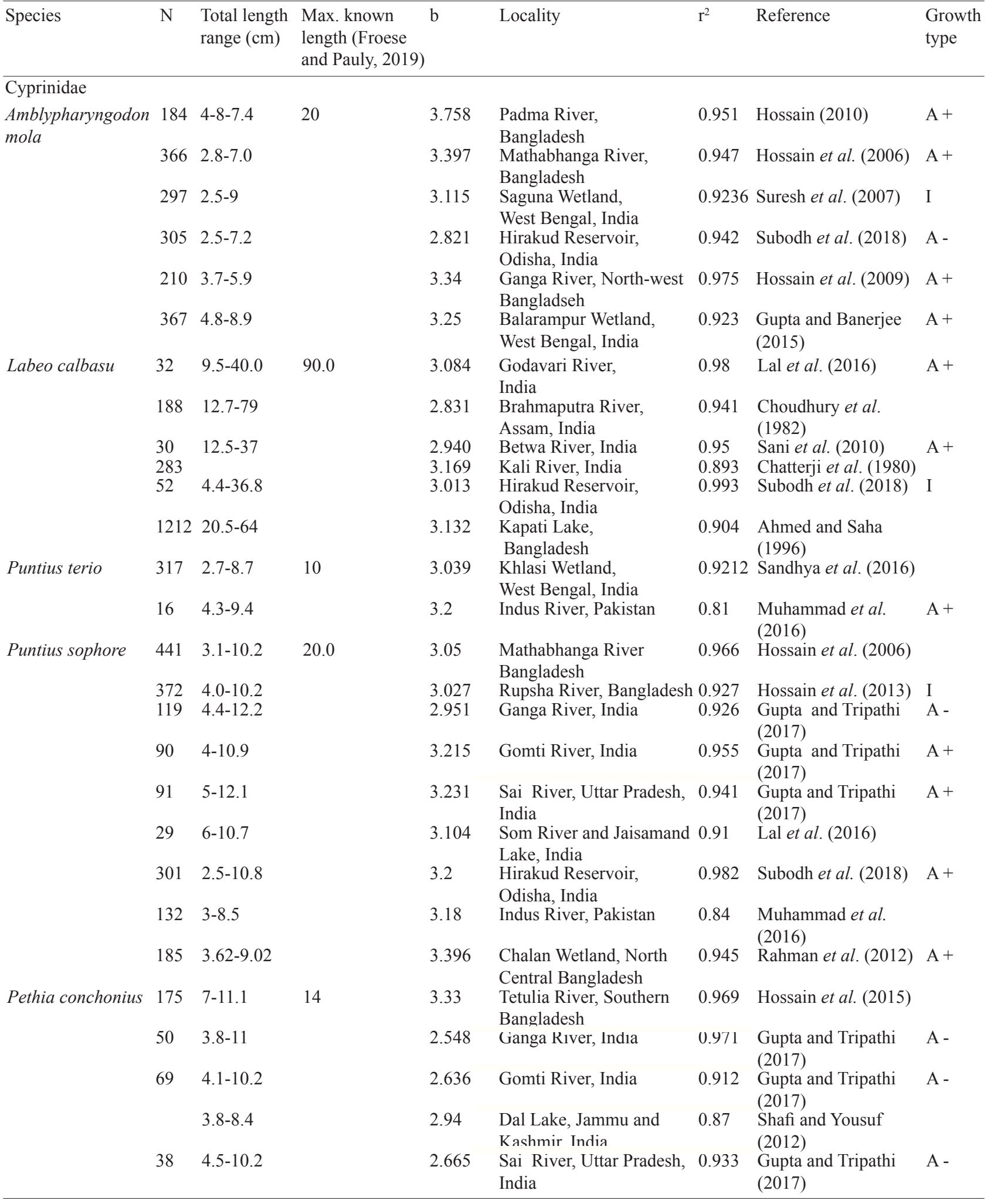




\begin{tabular}{|c|c|c|c|c|c|c|c|c|}
\hline Species & $\mathrm{N}$ & $\begin{array}{l}\text { Total length } \\
\text { Range }(\mathrm{cm})\end{array}$ & $\begin{array}{l}\text { Max known } \\
\text { length (Froese } \\
\text { and Pauly, 2019) }\end{array}$ & $\mathrm{b}$ & Locality & $\mathrm{r}^{2}$ & Reference & $\begin{array}{l}\text { Growth } \\
\text { type }\end{array}$ \\
\hline \multirow[t]{3}{*}{ Pethia phutunio } & 30 & $1.8-3.9$ & 3.9 & 2.82 & $\begin{array}{l}\text { Gajner Floodplain } \\
\text { Wetland, Bangladesh }\end{array}$ & 0.98 & Hossen et al. (2017) & \\
\hline & 117 & $1.98-3.3$ & & 2.8512 & Khalsi Wetlnd, India & 0.9220 & Sandhya et al. (2016) & \\
\hline & 96 & $1.9-3.7$ & & 2.623 & $\begin{array}{l}\text { Hirakud Reservoir, } \\
\text { Odisha, India }\end{array}$ & 0.937 & Subodh et al. (2018) & A - \\
\hline \multirow[t]{4}{*}{$\begin{array}{l}\text { Ambassidae } \\
\text { Chanda nama }\end{array}$} & 443 & $1.6-10.10$ & & 2.858 & $\begin{array}{l}\text { Hirakud Reservoir, } \\
\text { Odisha, India }\end{array}$ & 0.98 & Subodh et al. (2018) & A - \\
\hline & & $4.5-6.8$ & & 2.845 & $\begin{array}{l}\text { Pagla River, } \\
\text { Bangladesh }\end{array}$ & 0.933 & Alam et al. (2013) & \\
\hline & 146 & $2.5-7$ & & 2.799 & $\begin{array}{l}\text { Deepor Wetland, } \\
\text { Assam. India }\end{array}$ & 0.94 & Borah et al. (2017) & \\
\hline & 43 & $4-7.4$ & & 2.869 & $\begin{array}{l}\text { Bhramaputra River, } \\
\text { Bangladesh }\end{array}$ & 0.963 & Islam et al. (2017) & \\
\hline \multirow[t]{3}{*}{$\begin{array}{l}\text { Parambassis } \\
\text { lala }\end{array}$} & 193 & $1.6-3.5$ & & 2.864 & $\begin{array}{l}\text { Hirakud Reservoir, } \\
\text { Odisha, India }\end{array}$ & 0.918 & Subodh et al. (2018) & A - \\
\hline & 148 & $2.23-3.55$ & & 2.7026 & $\begin{array}{l}\text { Khalsi Wetland, } \\
\text { West Bengal, } \\
\text { India }\end{array}$ & .9013 & Sandhya et al. (2016) & \\
\hline & 101 & $2.5-4$ & & 3.020 & $\begin{array}{l}\text { Deepor Wetland, } \\
\text { Assam, India }\end{array}$ & 0.88 & Borah et al. (2017) & \\
\hline \multirow[t]{3}{*}{$\begin{array}{l}\text { Parambassis } \\
\text { ranga }\end{array}$} & 330 & $1.6-8.2$ & & 2.794 & $\begin{array}{l}\text { Hirakud Reservoir, } \\
\text { Odisha, India }\end{array}$ & 0.973 & Subodh et al. (2018) & A - \\
\hline & 148 & $2.23-3.55$ & & 2.7026 & Khalsi Wetland, India & 0.9013 & Sandhya et al. (2016) & \\
\hline & 595 & $2-5.2$ & & 2.67 & $\begin{array}{l}\text { East Kolkata Wetlands, } \\
\text { West Bengal, India }\end{array}$ & 0.857 & Mahaptra et al. (2014) & A - \\
\hline \multirow{4}{*}{$\begin{array}{l}\text { Bagridae } \\
\text { Sperata } \\
\text { seenghala }\end{array}$} & 131 & $46-113$ & & 3.05 & Indus River, Pakistan & 0.99 & Jatoi et al. (2013) & I \\
\hline & 205 & $40-115$ & & 2.866 & Ganga River, India & 0.95 & Khan et al. (2011) & A - \\
\hline & 20 & $21.0-68.0$ & 150.0 & 3.302 & $\begin{array}{l}\text { Som River and } \mathrm{J} \\
\text { aisamand Lake, India }\end{array}$ & 0.99 & Lal et al. (2016) & \\
\hline & 92 & $20-67$ & & 3.07 & Gomti River, India & 0.93 & Sarkar et al. (2013) & \\
\hline Sperata aor & $\begin{array}{l}184 \\
300\end{array}$ & $\begin{array}{l}72-95 \\
29-68\end{array}$ & 180 & $\begin{array}{l}3.249 \\
3.006\end{array}$ & $\begin{array}{l}\text { Ganga River, India } \\
\text { Nagarjuna Sagar, } \\
\text { Andhra Pradesh, India }\end{array}$ & 0.98 & $\begin{array}{l}\text { Khan et al. (2011) } \\
\text { Ramkrishniah (1998) }\end{array}$ & $\mathrm{A}+$ \\
\hline \multirow[t]{4}{*}{$\begin{array}{l}\text { Glossogobius } \\
\text { giuris }\end{array}$} & $\begin{array}{l}30 \\
159\end{array}$ & $\begin{array}{l}12.4-45 \\
8.7-17.9\end{array}$ & & $\begin{array}{l}2.98 \\
3.03\end{array}$ & $\begin{array}{l}\text { Betwa River, India } \\
\text { Ganga River, North-west } \\
\text { Bangladesh }\end{array}$ & $\begin{array}{l}0.97 \\
0.958\end{array}$ & $\begin{array}{l}\text { Sani et al. (2010) } \\
\text { Hossain et al. (2009) }\end{array}$ & \\
\hline & 129 & $2.3-22.5$ & & 2.914 & $\begin{array}{l}\text { Hirakud Reservoir, Odisha, } \\
\text { India }\end{array}$ & 0.98 & Subodh et al. (2018) & A - \\
\hline & 31 & $8.4-27.0$ & 50.0 & 2.974 & Betwa River, India & 0.98 & Lal et al. (2016) & \\
\hline & 49 & $5.5-9.7$ & & 2.682 & $\begin{array}{l}\text { Bhramaputra River, } \\
\text { Bangladesh }\end{array}$ & 0.867 & Islam et al. (2017) & A - \\
\hline \multirow[t]{2}{*}{$\begin{array}{l}\text { Schilbeidae } \\
\text { Ailia coila }\end{array}$} & 103 & $6.6-13$ & & 3.01 & $\begin{array}{l}\text { Ganga, North-west } \\
\text { Bangladesh }\end{array}$ & 0.981 & Hossain et al. (2009) & \\
\hline & 105 & $8.1-15.6$ & & 3.076 & $\begin{array}{l}\text { Padma River, North-west } \\
\text { Bangladesh }\end{array}$ & 0.986 & Hossain (2010) & I \\
\hline
\end{tabular}




\begin{tabular}{|c|c|c|c|c|c|c|c|c|}
\hline Species & $\mathrm{N}$ & $\begin{array}{l}\text { Total length } \\
\text { Range }(\mathrm{cm})\end{array}$ & $\begin{array}{l}\text { Max known } \\
\text { length (Froese } \\
\text { and Pauly, 2019) }\end{array}$ & $\mathrm{b}$ & Locality & $\mathrm{r}^{2}$ & Reference & $\begin{array}{l}\text { Growth } \\
\text { type }\end{array}$ \\
\hline \multirow[t]{3}{*}{$\begin{array}{l}\text { Cichlidae } \\
\text { Oreochromis } \\
\text { niloticus }\end{array}$} & 189 & $9.7-16$ & & 2.313 & $\begin{array}{l}\text { Barur Reservoir, } \\
\text { Tamil Nadu, India }\end{array}$ & & Marx et al. (2014) & \\
\hline & $\begin{array}{l}240 \\
575\end{array}$ & $6.9-27.3$ & & $\begin{array}{l}3.073 \\
3.08\end{array}$ & $\begin{array}{l}\text { White Nile River, Sudan } \\
\text { Wadi Hanifah Valley, } \\
\text { Saudi Arabia }\end{array}$ & $\begin{array}{l}0.9626 \\
0.96\end{array}$ & $\begin{array}{l}\text { Karrar et al. (2016) } \\
\text { Mortuza and Misned } \\
\text { (2013) }\end{array}$ & $\begin{array}{l}\mathrm{A}+ \\
\mathrm{A}+\end{array}$ \\
\hline & 4676 & $4.1-26.1$ & & $\begin{array}{l}3.43 \\
2.8006\end{array}$ & $\begin{array}{l}\text { ERO Reservoir, Nigeria } \\
\text { El-Faraouny Canal, } \\
\text { Al-Minufiya Province, } \\
\text { Egypt }\end{array}$ & $\begin{array}{l}0.83 \\
0.956\end{array}$ & $\begin{array}{l}\text { Adebola et al. (2016) } \\
\text { El- Kashief } \\
\text { et al. }(2015)\end{array}$ & $\begin{array}{l}\mathrm{A}+ \\
\mathrm{A}-\end{array}$ \\
\hline \multirow[t]{2}{*}{$\begin{array}{l}\text { Notopteridae } \\
\text { Notopterus } \\
\text { notopterus }\end{array}$} & 300 & $8.1-36.0$ & 60.0 & 2.9015 & $\begin{array}{l}\text { Tilaiya Reservoir, } \\
\text { Jharkhand, India }\end{array}$ & 0.93 & Khan (2003) & \\
\hline & $\begin{array}{l}65 \\
76\end{array}$ & $\begin{array}{l}12-33 \\
7.8-30.1\end{array}$ & & $\begin{array}{l}3.326 \\
3.368\end{array}$ & $\begin{array}{l}\text { Godavari River, India } \\
\text { Hirakud Reservoir, } \\
\text { Odisha, India }\end{array}$ & $\begin{array}{l}0.98 \\
0.98\end{array}$ & $\begin{array}{l}\text { Lal et al. (2016) } \\
\text { Subodh et al. (2018) }\end{array}$ & $\mathrm{A}+$ \\
\hline \multicolumn{9}{|l|}{ Osphronemidae } \\
\hline \multirow{5}{*}{$\begin{array}{l}\text { Trichogaster } \\
\text { chuna } \\
\text { Siluridae } \\
\text { Ompok } \\
\text { bimaculatus }\end{array}$} & 115 & $2.6-6.3$ & & 3.215 & $\begin{array}{l}\text { Deepor Wetland, } \\
\text { Assam, India }\end{array}$ & 0.96 & Borah et al. (2017) & \\
\hline & 70 & $10.5-31.5$ & 45.0 & 3.218 & Godavari River, India & 0.98 & Lal et al. (2016) & \\
\hline & 44 & $16.0-43.0$ & 45.0 & 3.027 & $\begin{array}{l}\text { Sonriver and Jaisalmer } \\
\text { Lake, India }\end{array}$ & 0.94 & Lal et al. (2016) & \\
\hline & 314 & $21.1-31.5$ & & 2.778 & $\begin{array}{l}\text { Bhavanisagar Reservoir, } \\
\text { Tamil Nadu, India }\end{array}$ & 0.989 & Sivakami (1987) & A - \\
\hline & 42 & $13-34.7$ & & 3.117 & $\begin{array}{l}\text { Hirakud Reservoir, } \\
\text { Odisha, India }\end{array}$ & 0.98 & Subodh et al. (2018) & I \\
\hline \multirow{6}{*}{$\begin{array}{l}\text { Channidae } \\
\text { Channa } \\
\text { punctata }\end{array}$} & 284 & $16.8-29.8$ & & 3.12 & Ganga River, India & 0.94 & Khan et al. (2011) & $\mathrm{A}+$ \\
\hline & 355 & $6-18.90$ & & 3.037 & $\begin{array}{l}\text { Mathabhanga River, } \\
\text { Bangladesh }\end{array}$ & 0.979 & Hossain et al. (2006) & $A+$ \\
\hline & 200 & $15-30$ & & 2.621 & $\begin{array}{l}\text { Hussain Sagar Lake, } \\
\text { Andhra Pradesh, India }\end{array}$ & 0.991 & $\begin{array}{l}\text { Kumari and Kumar } \\
\text { (2015) }\end{array}$ & \\
\hline & 140 & & & 3.01 & $\begin{array}{l}\text { Gomti River, Lucknow, } \\
\text { India }\end{array}$ & 0.95 & Kashyap et al. (2014) & I \\
\hline & 17 & $12.0-21.5$ & 31.0 & 2.786 & $\begin{array}{l}\text { Som River and Jaisamand } \\
\text { Lake, India }\end{array}$ & 0.91 & Lal et al. (2016) & \\
\hline & 42 & $13-34.7$ & & 3.117 & $\begin{array}{l}\text { Hirakud Reservoir, } \\
\text { Odisha, India }\end{array}$ & 0.98 & Subodh et al. (2018) & $\mathrm{I}$ \\
\hline
\end{tabular}

population is often required for estimation of population size of a fish stock for the purpose of its rational exploitation (Dulcic and Kraljevic, 1996). Therefore the results from the present study could contribute to the knowledge of fish populations in this area and provide an important baseline for future studies in Panchet Reservoir in the Damodar River basin. The information generated from the study will be useful for formulating effective fisheries management strategies and in implementing regulations for conservation of the native fish stock.

\section{Acknowledgments}

The authors are grateful to Indian Ccouncil of Agricultural Research, New Delhi for funding support and the Director, ICAR-CIFRI, Barrackpore, India for his immense support, guidance and providing facilities for conducting the research. The support rendered by the Director and staff of the Department of Fisheries, Govt. of Jharkhand and the fishing community of Panchet Reservoir is duly acknowledged.

\section{References}

Adebola, O. T., Bello-Olusoji, O. A., Fagbenro, O. A. and Sabejeje, T. A. 2016. Length-weight relationship and condition factor of four commercially important fish species at Ero Reservoir, Ekiti State, Nigeria. Int. J. Innov. Res. Dev., 5(9): 324-328. 
Ahmed, K. K. and Saha, S. B. 1996. Length-weight relationships of major carps in Kaptai Lake, Bangladesh. Naga, ICLARM Q., 19(2), 28 .

Alam, M. M., Rahman, M. T. and Parween, S. 2013. Morphometric characters and condition factors of five freshwater fishes from Pagla River of Bangladesh. Int. J. Aquat. Biol., 19(2):14-19.

Bagenal, T. B. and Tesch, F. W. 1978. Age and growth. In: Bagenal T. (Ed.), Methods for assessment of fish production in freshwater, IBP Handbook 3. Blackwell Scientific Publications, Oxford, UK, p. 101-136

Basusta, A., Basusta, N., Ozer, E. I., Aslan, E. and Girgin, H. 2013. Some population parameters of the Lessepsian Suez Puffer (Lagocephalus suezensis) from Iskenderun Bay, North-eastern Mediterranean, Turkey. Pakistan. J. Zool., 45(6): 1779-1782.

Bolger, T. and Connolly, P. L. 1989. The selection of suitable indices for the measurement and analysis of fish condition. J. Fish. Biol., 34: 171-182. DOI: 10.1111/j.10958649.1989.tb03300.x.

Borah, S., Bhattacharjya, B. K., Saud, B. J., Yadav, A. K., Debnath, D., Yengkokpam, S., Das, P., Sharma, N., Singh, N. S. and Sarma, K. K. 2017. Length-weight relationship of six indigenous fish species from Deepor Beel, a Ramsar site in Assam, India. J. Appl. Icthyol., 33(3): 655-657. https://doi.org/ 10.1111/jai.13348.

Chatterji, A., Ali, M. and Mumtaz, A. 1980. A note on the lengthweight relationship of kalbasu, Labeo calbasu (Ham.). Indian. J. Fish., 27(1-2): 261-263.

Choudhury, M., Kolekar, V. and Chandra, R. 1982. Lengthweight relationship and relative condition factor of four Indian major carps of river Brahmaputra, Assam. J. Inland Fish. Soc. India, 14(2): 42-48.

Dulcic, J. and Kraljevic, M. 1996. Weight-length relationships for 40 fish species in the eastern Adriatic (Croatian waters). Fish. Res., 23: 243-251.

El-Kasheif, M. A., Mohammad, M. N., Al-Ghamdi, A. F. A., Ibrahim S. A. and El-Far, A. M. 2015. Biological aspects and fisheries management of tilapia fish Oreochromis niloticus (Linnaeus, 1758) in El-Bahr El-Faraouny Canal, Al-Minufiya Province, Egypt. Egypt. J. Fish. Aquat. Sci., 10(6): 405-444. DOI: $10.3923 /$ jfas.2015.405.444.

Froese, R., 2006. Cube law, condition factor and weight-length relationships: history, meta-analysis and recommendations. J. Appl. Ichthyol., 22(4): 241-253. https://doi.org/10.1111/j. 1439-0426.2006. 00805.x.

Froese, R. and Pauly, D. 2019. Fish Base. World Wide Web electronic publication. http://www. fishbase.org (Accessed 5 January 2019).

Goncalves, J. M. S., Bentes, L., Lino, P. G., Ribeiro, J., Canario, A. V. M. and Erzini, K. 1997. Weight-length relationship for selected fish species of the small scale demersal fisheries of the south and south-west coast of Portugal. Fish. Res., 30(3): 253-256. https://doi.org/10.1016/S01657836(96)00569-3.
Gupta, D. and Tripathi, M. 2017. Length-weight relationships and condition factors of five cyprinidae species (SubfamilyBarbinae) from three diverse rivers of Uttar Pradesh, India. Int. J. Fish. Aquat. Stud., 5(2): 594-598.

Gupta, S. and Banerjee, S. 2015. Length-weight relationship of Amblypharyngodon mola (HamBuch, 1822), a freshwater cyprinid fish from West Bengal, India. Zool. Ecol., 25: 54-58. DOI: 10.1080/ 21658005.2014.991513.

Hassan, M. A., Mishal, P., Karnatak, G. and Sharma, A. P. 2017. Towards the blue revolution in India: prospects for inland open waters. World. Aquac., 48(1): 25-28.

Hossain, M. Y., Ahmed, Z. F., Leunda, P. M., Islam, A. K. M. R., Jasmine, S., Oscoz, J., Miranda, R. and Ohtomi, J. 2006. Length-weight and length-length relationships of some small indigenous fish species from the Mathabhanga River, south-western Bangladesh. J. Appl. Ichthyol., 22(4): 301303. https:// doi.org/10.1111/j.1439-0426.2006.00801.x.

Hossain, M. Y., Ohtomi, J., Ahmed, Z. F., Ibrahim, A. H. M. and Jasmine, S. 2009. Length-weight and morphometric relationships of the tank goby Glossogobius giuris (Hamilton, 1822) (Perciformes: Gobiidae) in the Ganges of the North-western Bangladesh. Asian. Fish. Sci., 22(3): 961-969.

Hossain, M. Y. 2010. Morphometric relationships of lengthweight and length-length of four cyprinid small indigenous fish species from the Padma River (NW Bangladesh). Turk. J. Fish. Aquat. Sci., 10: 131-134. DOI: 10.4194/ trjfas.2010.0118.

Hossain, M. Y., Ohtomi, J., Jaman, A., Jasmine, S. and Vadas, R. L. Jr. 2012. Life-history traits of the monsoon river prawn Macrobrachium malcolmsonii (Milne-Edwards, 1844) (Palaemonidae) in the Ganges (Padma) River, northwestern Bangladesh. J. Freshw. Ecol., 27(1): 131-142. https://doi.org/ 10.1080/02705060.2011.633739.

Hossain, M. Y., Mosaddequr, M. R., Elgorban, M. A. and Jun, O. 2013. Biometric relationships of the pool barb Puntius sophore (Hamilton 1822) (Cyprinidae) from three major rivers of Bangladesh. Sains Malays., 42(11): 1571-1580.

Hossain, M. Y., Sayed, S. R. M., Rahman, M. M., Ali, M. M., Hossen, M. A., Elgorban, A. M., Ahmed, Z. F. and Ohtomi, J. 2015. Length-weight relationships of nine fish species from the Tetulia River, Southern Bangladesh. J. Appl. Ichthyol., 31(5): 967-969. DOI: 10.1111/jai.12823.

Hossain, M. Y., Hossen, M. A., Ahmed, Z. F., Hossain, M. A., Pramanik, M. N. U., Nawer, F., Paul, A. K., Khatun, D., Haque, N. and Islam, M. A. 2017. Length-weight relationships of 12 indigenous fish species in the Gajner Beel floodplain (NW Bangladesh). J. Appl. Ichthyol., 33: 842-845. https:// doi.org/10.1111/jai.13354.

Islam, M. R., Azom, G. M., Faridullah, M. and Mamun, M. 2017 Length-weight relationship and condition factor of 13 fish species collected from the Atrai and Brahmaputra rivers, Bangladesh. J. Biodivers. Environ. Sci., 10(3): 123-133. 
IUCN 2017. The IUCN red list of threatened species. Version 2017-3. http://www.iucnredlist.org. (Accessed 15 December 2017).

Jatoi, S., Baloch, W. A., Soomro, A. N. and Gachal, G. S. 2013. Length-weight relationship of the silurid catfish Sperata seenghala Sykes 1839 (Bagridae) from Indus River, Sindh, Pakistan. Sindh. Univ. Res. J., 45(4): 661-664.

Karrar, A. M. H. M., Elkareem, M. M. E. A. and Ali, A. K. S. 2016. Length-weight relationship and condition factor of Nile tilapia [Oreochromis niloticus (Trewavas)] from White Nile, Sudan. Environ. Nat. Res. Int. J., 1(1): 77-84.

Kashyap, A., Awasthi, M. and Serajuddin, M. 2014. Lengthweight and length-length relationship of freshwater murrel, Channa punctatus (Bloch, 1793) sampled from river Gomti in Lucknow region (Uttar Pradesh). World. J. Fish. Mari. Sci., 6(4): 336-339. DOI: 10.5829/idosi. wjfms.2014.06.04.84293.

Khan, M. A. 2003. Length-weight relationship and relative condition index of Notopterus notopterus (pallas) of Tilaiya Reservoir, Bihar. Records Zool. Surv. India, 101(3-4): 233-239.

Khan, S., Khan, M. A., Miyan, K. and Mubarak, M. 2011. Length-weight relationships for nine freshwater teleosts collected from river Ganga. Int. J. Zool. Res., 7: 401-405. DOI: 10.3923/ijzr.2011.401.405.

King, R. P. 1996. Length-weight relationships and related statistics of 73 populations of fish occurring in inland waters of Nigeria. NAGA, ICLARM Q., 19: 49-52.

Kumari, S. A. and Kumar, N. S. R. 2015. Length-weight relationship and condition factor in Channa punctatus (Bloch) from Hussain Sagar Lake, Hyderabad, Andhra Pradesh, India. In: Rawat, M., Dookia S. and Sivaperuman C. (Eds.), Aquatic ecosystem: Biodiversity, ecology and conservation, Springer, New Delhi, p. 283-286.

Lal, K. K., Gupta, B. K., Dwivedi, A. K., Singh, R. K., Chandra, S. and Mohindra, V. 2016. Length-weight relationship of selected freshwater fish species from three diverse ecological regimes in India. Indian. J. Fish., 63(1): 107-112.

Le Cren, E. D. 1951. The length-weight relationship and seasonal cycle in gonad weight and condition in the perch (Perca fluviatilis). J. Anim. Ecol., 20(2): 201-219. DOI: $10.2307 / 1540$.

Lianthuamluaia, L., Mishal, P., Panda, D., Sarkar,U.K., Kumar, V., Sandhya, K. M., Karnatak, G., Kumari, S., Bera, A. K., Das, S. and Ali, Y. 2019. Understanding spatial and temporal patterns of fish diversity and assemblage structure vis-a-vis environmental parameters in a tropical Indian reservoir. Environ. Sci. Pollut. Res., 26(9): 9089-9098. DOI:10.1007/s11356-019-04305-

Mahapatra, B. K., Pal, M., Bhattacharjee, S. and Lakra, W. S. 2014. Length-weight relationship and condition factor of an indigenous ornamental fish, Pseudambassis ranga (Hamilton, 1822) from East Kolkata Wetland. Int. J. Fish. Aquat. Stud., 2(2): 173-176.
Marx, K. K., Vaitheeswaran, T., Chidambaram, P., Sankarram, S. and Karthiga, P. 2014. Length-weight relationship of Nile tilapia of Oreochromis niloticus (Linnaeus, 1758) (Family: Cichlidae). Int. J. Fish. Aquat. Stud., 2(2): 173-176.

Mortuza, M. G. and Al-Misned, F. A. 2013. Length-weight relationships, condition factor and sex-ratio of Nile tilapia, Oreochromis niloticus in Wadi Hanifah, Riyadh, Saudi Arabia. World. J. Zool., 8(1): 106-109. DOI: 10.5829/idosi. wjz.2013.8.1.7247.

Muhammad, H., Iqbal, Z. and Akhlaq, T. 2016. Length-weight, length-length relationships and condition factor of fishes of family Cyprindae from the Indus River, Pakistan. Punjab. Univ. J. Zool., 31(2): 143-147.

Oscoz, J., Campos F. and Escala, M. C. 2005. Weight-length relationships of some fish species of the Iberian Peninsula. J. Appl. Ichthyol., 21(1): 73-74. https://doi.org/10.1111/ j.1439-0426.2004.00587.x

Pauly, D., Gayanilo, Jr., F. C. 1997. ABee: An alternative approach to estimating the parameters of a length-weight relationship from length-frequency samples and their bulk weights. International Centre for Living Aquatic Resources Management, Manila, Philippines.

Petrakis, G. and Stergiou, K. I. 1995. Weight-length relationships for 33 fish species in Greek waters. Fish. Res., 21: 465-469. https://doi.org/10.1016/0165-7836(94)00294-7.

Pinheiro, A. A. M. and Fiscarelli, A. G. 2009. Length-weight relationship and condition factor of the mangrove crab Ucides cordatus (Linnaeus, 1763) (Crustacea, Brachyura, Ucididae). Brazilian. Arch. Biol. Technol., 52(2): 397-406. https://doi.org/10.1590/S1516-89132009000200017.

Rahman, M. M., Hossain, M. Y., Jewel, M. B. S., Mohammad, M. R., Jasmine, S., Elgorban, M. A. and Ohtomi, J. 2012. Sains. Malays., 41: 795-802.

Ramakrishniah, M. 1998. Age, growth and fishery of Mystus aor (Hamilton) from Nagarjunasagar Reservoir. In: Mohan Joseph, M. (Ed.), Proceedings of the First Indian Fisheries Forum, Asian Fisheries Society, Indian Branch, Mangalore, Karnataka, India, p 185-189.

Richter, H., Luckstadt, C., Focken, U. and Becker, K. 2000. An improved procedure to assess fish condition on the basis of length-weight relationships. Arch. Fish. Mar. Res., 48(3): 255-264.

Ricker, W. E. 1973. Linear regressions in fishery research. Fish. Res. Board Can. Bull., 30(3): 309-434.

Sandhya, K. M., Hassan, M. A., Kumari, S., Mishal, P., Lianthuamluaia, L., Kumar, V., Aftabuddin, M. and Meena, D. K. 2016. Length-weight relationships of four indigenous freshwater fish species from Khalsi wetland in lower Ganga Basin, West Bengal, India. J. Appl. Ichthyol., 32: $505-506$

Sandhya, K. M., Sarkar, U. K., Karnatak, G., Lianthuamluaia, L., Kumar, V., Kumari, S., Panda, D., Mishal, P. and Ali, Y. 2017. Length weight relationships of two small indigenous cyprinid fishes, Osteobrama cotio (Hamilton, 1822) 
and Salmophasia phulo (Hamilton, 1822) from Panchet Reservoir, Damodar River (tributary of River Ganga), India. J. Appl. Ichthyol., 33(3): 635-636. https://doi. org/10.1111/ jai.13328.

Sandhya, K. M., Lianthuamluaia, L., Karnatak, G., Sarkar, U. K., Kumari, S., Mishal, P., Kumar, V., Panda, D., Ali, Y. and Naskar, B. K. 2019. Fish assemblage structure and spatial gradients of diversity in a large tropical reservoir, Panchet in the Ganges Basin, India. Env. Sci. Pollut. Res., 26: 18804-18813. doi: 10.1007/s11356-019-05314-8.

Sani, R., Gupta, B. K., Sarkar, U. K., Pandey, A., Dubey, V. K. and Lakra, W. S. 2010. Length-weight relationship of 14 Indian freshwater fish species from the Betwa (Yamuna River tributary) and Gomti (Ganga River tributary) rivers. J. Appl. Ichthyol., 26(3): 456-459. https://doi.org/10.1111/ j.1439-0426.2009.01388.x.

Sarkar, L. and Banerjee, S. 2010. Breeding ground profile of food fish species in Damodar River system. Int. J. Biol., 2(1): 51-60

Sarkar, U. K., Khan, G. E., Dabas, A., Pathak, A. K., Mir, J. I., Rebello, S. C., Pal, A. and Singh S. P. 2013. Length-weight relationship and condition factor of selected freshwater fish species found in River Ganga, Gomti and Rapti, India. J. Environ. Biol., 34(5): 951-956.

Sarkar, U. K. and Mishal, P. 2017. Status, potentials and prospects of reservoir and wetland fisheries in India - an overview. In: Mohanty et al. (Eds.), Souvenir, National Seminar on Priorities in Fisheries and Aquaculture (PFA 2017), 11-12 March 2017. ICAR-Central Inland Fisheries Research Institute, Barrackpore, India, p. 171-185.

Sarkar, U. K., Sandhya, K. M., Mishal, P., Karnatak, G., Lianthuamluaia,L., Kumari,S.,Panikkar,P.,Palaniswamy,R., Karthikeyan, M., Sibinamol, S., Paul, T. T., Ramya, V. L.,
Rao, D. S. K., Khan, M. F., Panda, D. and Das, B. K. 2018. Status, prospects, threats and the way forward for sustainable management and enhancement of the tropical Indian reservoir fisheries: an overview. Rev. Fish. Sci. Aquac., 26(20): 155-175.

Shafi, S. and Yousuf, A. R. 2012. Length-weight relationship and condition factor in Puntius conchonius (Hamilton, 1822) from Dal Lake, Kashmir. Int. J. Sci. Res. Publ., 2(3): 1-4.

Sivakami, S. 1987. Length-weight relationship and relative condition in Ompok bimaculatus (Bloch) from Bhavanisagar Reservoir (Tamil Nadu). Indian J. Fish., 34(2): 202-207.

Sokal, R. R. and Rohlf, F. J. 1987. Introduction to biostatistics, $2^{\text {nd }}$ edn. Freeman Publication, New York, USA.

Subodh, K. K., George, N. K. and Laith, A. J. 2018. Lengthweight relations of 24 fish species (Actinopterygii) from Hirakud Reservoir, Odisha State of India. Acta. Ichthyol. Piscat., 48(1):83-86.

Suman, K., Sarkar, U. K., Sandhya, K. M., Lianthuamluaia, L., Panda, D., Chakraborty, S. K., Gunjan, K., Vikash, K. and Mishal, P. 2018. Studies on the growth and mortality of Indian River shad, Gudusia chapra (Hamilton, 1822) from Panchet Reservoir, India. Environ. Sci. Pollut. Res., 25(33):33768-33772.

Suresh, V. R., Biswas, B. K., Vinci, G. K., Mitra, K. and Mukherjee, A. 2007. Biology of Amblypharyngodon mola (Hamilton) from a floodplain wetland, West Bengal. Ind. J. Fish., 54(2): 155-161.

Weatherley, A. H. and Gill, H. S. 1987. The biology of fish growth. Academic Press, London, UK.

Wootton, R. J. 1999. Ecology of teleost fishes. Chapman and Hall, London, UK. 